\title{
Dark matter and the deformed Poincaré group
}

\author{
J. Fernandez ${ }^{1}$ \\ Departamento de Física, Universidad Nacional de La Plata, C.C. 67, 1900 La Plata, Argentina
}

Received 22 September 1995

Editor: M. Dine

\begin{abstract}
It has been recently suggested that models of deformed special relativity, based on a deformed Poincaré group, could account for the missing mass problem, due to the nonadditivity of energy for large systems predicted by these models. We show that this is not correct and that, in fact, these models predict just the opposite effect.
\end{abstract}

Recently, different explanations have been proposed to account for the missing mass problem. These explanations range from the existence of invisible brown dwarf stars (a "conservative" explanation) or the existence of a new kind of matter filling the universe (cold nonbaryonic dark matter), until more radical explanations proposing the need of new laws of physics [1].

The missing mass problem arises when astronomers look at the rotation curves of galaxies (i.e. the pattern of the velocities of stars around a galaxy). They find that, in order to get the galaxies clumped, it would be necessary to have much more mass inside them than observed. The "observed" mass refers to the estimated mass of galaxies obtained counting the "visible" mass (stars, HII regions, molecular gas clouds, etc.). Thus, we have an "observed" mass and a "dynamical" mass (the latter is obtained assuming the approximate validity of Newton's gravitational laws). The same happens with galaxies inside clusters of galaxies.

The dynamical mass is of the order of 10-100 times the observed mass. Thus, a lot of research has been devoted in order to account for the missing mass. In par-

'E-mail: fernande@venus.fisica.unlp.edu.ar. ticular, models of "deformed" special relativity, based on a deformed Poincaré Group [2-6] show that energy is not additive for large systems in these models, this means that the total energy of the universe would not be proportional to the number of particles it contains. This implies that for large systems, the mass "observed" will differ from the real mass.

We will not enter the details of the theory of deformations of the Poincaré group. In Ref. [7] all physically acceptable deformed Poincaré algebras are classified and the advantages of such deformations are resumed.

Recently, Maślanka [8] proposed a parametrization of a family of deformed Poincaré groups which has been used by Bacry [6] to study the nonadditivity of energy and to suggest that this could account for the missing mass ( so that there would be no dark matter to discover). We will show that this family of deformed Poincaré groups is not useful in explaining a mass defect. In fact, it predicts the opposite observed effect, posing doubts about its validity as a model of the real physical world.

If we denote by $\boldsymbol{J}, \boldsymbol{K}, \boldsymbol{P}$ and $P_{0}$ the generators of the ordinary Poincaré group (which generate rotations, 
boosts and space and time translations respectively), we can write the commutation relations in the following compact way:

$$
\begin{array}{lll}
{[J, J]=i J,} & {[J, K]=i K,} & {[J, P]=i P,} \\
{\left[J, P_{0}\right]=0,} & {[K, K]=-i J,} & {[K, P]=i P_{0},} \\
{\left[K, P_{0}\right]=i P,} & {[P, P]=0,} & {\left[P, P_{0}\right]=0 .}
\end{array}
$$

On the other hand, let $A(\kappa)$ denote the family of deformed algebras depending on the energy parameter $\kappa$. We denote the corresponding generators of $A(\kappa)$ by the same letters with subindices. Thus, the commutation relations for the $\boldsymbol{\kappa}$-deformed Poincare algebra reads

$$
\begin{aligned}
& {\left[J_{\kappa}, J_{\kappa}\right]=i J_{\kappa}, \quad\left[J_{\kappa}, K_{\kappa}\right]=i K_{\kappa},} \\
& {\left[J_{\kappa}, P_{\kappa}\right]=i P_{\kappa}, \quad\left[J_{\kappa}, P_{0 \kappa}\right]=0,} \\
& {\left[K_{\kappa}, K_{\kappa}\right]=-i \cosh \left(\frac{P_{0 \kappa}}{\kappa}\right) J_{\kappa}-\frac{1}{4 \kappa^{2}}\left(J_{\kappa} \cdot P_{\kappa}\right) P_{\kappa},} \\
& {\left[\boldsymbol{K}_{\kappa}, P_{\kappa}\right]=i \kappa \sinh \left(\frac{P_{0 \kappa}}{\kappa}\right), \quad\left[\boldsymbol{K}_{\kappa}, P_{0 \kappa}\right]=i P_{\kappa},} \\
& {\left[\boldsymbol{P}_{\kappa}, P_{\kappa}\right]=0, \quad\left[\boldsymbol{P}_{\kappa}, P_{0 \kappa}\right]=0 .}
\end{aligned}
$$

Then, the Maślanka mapping is the following one:

$$
\begin{aligned}
J_{\kappa} & =J, \quad P_{\kappa}=P, \\
P_{0 \kappa} & =2 \kappa \arg \sinh \frac{P_{0}}{2 \kappa}, \\
K_{\kappa} & =\frac{1}{2} \sqrt{1+\frac{P_{0}^{2}}{4 \kappa^{2}}} K \\
& +\frac{\sqrt{1+P_{0}^{2} / 4 \kappa^{2}}-\sqrt{1+m^{2} / 4 \kappa^{2}}}{P_{0}^{2}-m^{2}} \\
& \times P \times\left(P_{0} J-K \times P\right) .
\end{aligned}
$$

Among the new momenta, only $\boldsymbol{J}_{\boldsymbol{\kappa}}$ and $\boldsymbol{P}_{\boldsymbol{\kappa}}$ possess the additivity property; it means, for instance, that the momentum $\boldsymbol{P}$ of a system $\mathrm{S}$ composed of two noninteracting subsystems $S_{1}$ and $S_{2}$ with momenta $\boldsymbol{P}_{1}$ and $\boldsymbol{P}_{2}$ is the sum $\boldsymbol{P}_{1}+\boldsymbol{P}_{2}$. Although the energy of an isolated system is still conserved, the energy $P_{0 \kappa}$ of $S$ is no longer the sum of the energies of the two subsystems $S_{1}$ and $S_{2}$. Instead we have

$$
2 \kappa \sinh \frac{P_{0 \kappa}}{2 \kappa}=2 \kappa \sinh \frac{P_{0 \kappa}^{1}}{2 \kappa}+2 \kappa \sinh \frac{P_{0 \kappa}^{2}}{2 \kappa} .
$$

Bacry [6] interprets that this relation can explain the illusion of a missing mass in the universe. But we will see that this relation implies the opposite.

If wc consider a set of $N$ nucleons (the "real" nucleons, observed by counting the "visual" mass), the mass $M$ of such a set would be given by (from (1))

$\sinh \frac{M}{2 \kappa}=N \sinh \frac{m}{2 \kappa}$,

where $m$ is the mass of one nucleon. Because we have the illusion of additivity of the energy, we think of $M$ (which provokes the dynamical effects) as arising from $N_{\text {eff }}$ effective nucleons, with $N_{\text {eff }}=M / m$. Therefore, we have

$\sinh \frac{m N_{\mathrm{eff}}}{2 \kappa}=N \sinh \frac{m}{2 \kappa}$.

This equation implies that always $N_{\text {eff }}<N$, independently of $\kappa, m$ or $N$. The ratio $N / N_{\text {eff }}$ grows as $N$ grows. Thus, if we assume additivity of energy and calculate the "mass" from dynamical observations, we will conclude erroneously that there is less mass than visually observed. But as we had explained before, we observe the opposite effect: that there is more mass than visually observed.

Thus, we can conclude that models of deformed Poincaré group not only can not solve the missing mass problem but grows the things worse. So, we have to pose doubts about the validity of these models as a good description of the real physical world.

This work is supported by Universidad Nacional de La Plata (Argentina).

\section{References}

[11 J. Binney and S. Tremaine, Galactic Dynamics (Princeton University Press, 1987).

[2] W.B. Schmidke, J. Wess and B. Zumino, Z. Phys. C 52 (1991) 471; Nucl. Phys. B 18 (1990) 302

[3] O. Ogiewetsky, W.B. Schmidke, J. Wess and B. Zumino, Commun. Math. Phys. 150 (1992) 495.

[4] J. Lukierski, A. Nowicki, H. Ruegg and V.N. Tolstoy, Phys. Lett. B 264 ( 1991 ) 331.

[5] J. Lukierski, A. Nowicki and H. Ruegg, Phys. Lett. B 293 (1992) 344.

[6] H. Bacry, Phys. Lett. B 317 (1993) 523.

[7] H. Bacry, Which deformations of the Poincaré group?, Marseille preprint CPT-93/P.2880 (March 1993).

[8] P. Maślanka, Deformation map and hermitean representations of k-Poincaré algebra, Lodz preprint (April 1993). 